Article

\title{
Towards Management of Residual Limb Volume: Monitoring the Prosthetic Interface Pressure to Detect Volume Fluctuations-A Feasibility Study
}

\author{
Chakaveh Ahmadizadeh ${ }^{1,2}$ (D), Brittany Pousett ${ }^{3}\left(\mathbb{D}\right.$ and Carlo Menon ${ }^{1,2, *(D)}$ \\ 1 Menrva Research Group, Schools of Mechatronic Systems Engineering and Engineering Science, \\ Simon Fraser University (Burnaby Mountain Campus), Metro Vancouver, BC V5A 1S6, Canada; \\ cahmadiz@sfu.ca \\ 2 Menrva Research Group, Schools of Mechatronic Systems Engineering and Engineering Science, \\ Simon Fraser University (Surrey Campus), Metro Vancouver, BC V3T 0A3, Canada \\ 3 Barber Prosthetics Clinic, Vancouver, BC V5X 2T4, Canada; brittany@barberprosthetics.com \\ * Correspondence: cmenon@sfu.ca
}

Received: 24 August 2020; Accepted: 21 September 2020; Published: 29 September 2020

\begin{abstract}
Motivation: Variations in the volume of the residual limb negatively impact various aspects of prosthesis use including the prosthetic socket fit. Although volume adjustment systems mitigate corresponding fit problems to some extent, some users still find the management of these systems challenging. With the ultimate goal of creating a feedback system that assists users with the management of their volume adjustment systems, this study demonstrates the feasibility of detecting variations in the volume of the residual limb. (2) Methods: Measurements of the interface force at the bottom of the prosthetic socket were used as indicators of variations in the volume of the residual limb. Force sensitive resistors (FSRs) were placed at the bottom of participants' prosthetic sockets to monitor the interface limb-socket force as participants walked on a flat surface. Two phases of experiments were carried out: The first phase considered variations simulated by three prosthetic sock plies, established the feasibility of detecting variations in the volume of the limb based on the interface force, and further determined the locations at which the interface force could be used to detect variations in the limb's volume. Having validated the effectiveness of the proposed method in the first phase, the second phase was carried out to determine the smallest detectable variation of the limb's volume using the proposed method. In this phase, variations simulated by one and two prosthetic sock plies were considered. Four and three volunteers with transtibial amputations participated in the first and the second phases, respectively. (3) Results: Results of the first phase showed that an increase in the volume of the limb resulted in a decrease in the force measured at the distal location of the prosthetic sockets of all participants; however, the smallest detected variation could not be statistically confirmed.
\end{abstract}

Keywords: residual limb volume; prosthetic sock; distal pressure; sock management; socket fit; force sensitive resistor; volume fluctuation; sock ply

\section{Introduction}

This study proposes a technique that can be used by individuals with lower limb amputations for the management of their residual limb's volume variations, especially when using prosthetic socks. The focus of this study was to develop a low-complexity system such that it would be inexpensive, easy to install inside the user's prosthetic socket, less prone to failures, and easy to use from the end-user's perspective. These characteristics would increase the impact that this technology would have on the affected population by enhancing the adaptability of the developed system. 
Variations in the shape and volume of an individual's residual limb are common amongst individuals with amputations. Some of the causes of such variations are the residual limb's fluid volume changes, the user's weight variations, the limb's muscle activity, and muscle atrophy and edema in immature residual limbs-defined as less than a year post amputation [1,2]. Even in mature residual limbs, both diurnal and long term fluctuations in the volume of the limb may occur. The amount of volume variations in mature residual limbs varies considerably amongst individuals depending on their activity level, socket fit, dietary habits, etc. [3-5]. Although volume changes occur in both upper and lower extremities, due to the weight bearing nature of lower residual limbs, such volume fluctuations are more considerable in individuals with lower extremity amputations, which accounted for more than $65 \%$ of all amputations in the United States in 2005 [6].

Variations in the size of the limb can negatively impact the quality of the prosthetic socket fit, leading to the user's discomfort and causing skin health problems, such as edema, dermatitis, and ulceration [7-9] which are common problems in individuals with amputations of the lower limb.

A commonly prescribed volume management method to mitigate variations in the volume of the residual limb is to add or remove prosthetic sock plies worn on the limb $[2,4,10]$. Although prosthetic socks and other volume management methods are available and commonly used to compensate for volume fluctuations in the residual limb, management of sock plies remains an issue amongst users [3], especially those with cognitive issues and/or limited sensation in their residual limbs [11].

Various methods can be used to guide users in managing their volume adjustment systems to maintain a proper socket fit. Measurements of the volume of the limb can be used to provide feedback to assist users in maintaining a proper socket fit. The most practical volume measurement method for this application is bioimpedance that measures limb's volume by monitoring its resistance to electrical current which is then used to determine changes in fluid volume of the limb [12]. Although the bioimpedence method has shown promise for limb volume measurements within the socket, it requires electrodes to make proper contact with the skin through the use of hydrogel [13], which makes this method less favorable.

Another approach to address the problem considered in this study is to focus on biomechanical analysis, e.g., through development of a numerical model of the limb based on which the assessment of the positions and the number of required sensors can be determined. Although such methods have been explored in various articles for different applications including the assessment of the residual limb-socket interface pressure [14,15], a great amount of variability can be observed in the reported results and parameters used in such works. Due to such variability, results of these types of studies are often validated using experimental measurements [16-18]. In fact, one of the most common methods used to obtain experimental measurements of the interface pressure for validation of such biomechanical models is the use of force/pressure sensors [18-20]. Moreover, such a biomechanical model is individual-specific and based on measurements obtained from scans of each user, e.g., magnetic resonance imaging scans, to take into account the conditions of each individual's residual limb and prosthetic socket. Therefore, the generated biomechanical model would not be generalizable, making this method relatively complex and expensive.

Alternative methods using relatively accurate force sensors, such as load cells, for registering the pressure map at the interface of the prosthetic socket and the residual limb, which can be used towards the goal of this study, have also been investigated. Sanders et al. investigated this method in a study where they measured the interface pressure using 13 transducers mounted on the users' prosthetic sockets to investigate the interface pressures as socket fit was adjusted with fluid filled inserts [21]. Although the measurements obtained by such transducers can be used to determine and adjust the quality of the socket fit, these sensors are relatively expensive, not flexible, and difficult to incorporate in prosthetic sockets as modifications to the socket are required to mount the sensors. As reported in the study by Sanders et al., the transducers used in their investigations added $320 \mathrm{~g}$ to the system, which is another disadvantage of using such sensors for everyday use of this application. 
Force sensitive resistors (FSRs) are alternative solutions for prosthetic applications. The use of FSR-based sensory systems for acquisition of pressure maps at the interface of the residual limb and the prosthetic socket has been explored.Portnoy et al. investigated the use of FSRs for analysis of residual limb stresses [18]. In their study, measurements acquired from FSRs were used as boundary conditions for finite element analysis of stresses. Although this method utilized FSRs that are thin, flexible, easy to place inside the socket, and could potentially assist users with managing their limb volume adjustment system, it required additional data from $X$-rays and indentation tests to determine dimensions of the bones and soft tissues and elastic modulus of soft tissues, which makes the method relatively complex and costly.

The motivation behind this study was to develop a simple, inexpensive method that can determine fluctuations in the volume of the residual limb with the ultimate goal of using this information to assist users with the management of their volume adjustment system to prevent skin health problems that are caused by an inadequate fit of the prosthetic socket. Towards this goal, the study presented in this paper proposes an alternative method for determining variations in the volume of the limb through monitoring distal forces with a limited number of FSRs at the bottom of the socket. The proposed method is simple, easy to incorporate into the socket, and inexpensive. Furthermore, it does not require modifications to the socket and does not rely on electrical contact with the skin or information from $\mathrm{X}$-rays or indentation tests.

The study presented in this paper demonstrates the feasibility of detecting variations in the volume of the limb using the proposed method and identified the locations at the bottom of the socket at which the variations of pressure would be representative of the changes in the volume of the limb.

\section{Materials and Methods}

This study proposes a method that uses a simple hardware setup for the measurement of distal socket-limb interface force. Contrary to the FSR-based sensory systems used in other prosthetic applications, i.e., force myography (FMG) controlled prostheses and prosthetic socket fitting, this system uses a minimal number of FSRs to achieve its goal. The primary goal of this study was to evaluate if a simple technology based on the smallest possible number of FSRs could be used to provide feedback to the users for the management of volume adjustment systems, especially sock plies. As a result, prosthetic socks were added and removed for simulation of limb volume variations in order to assess the sensitivity of the method to these changes. To determine the feasibility of the proposed method, the correlation between variations in force measured by FSRs placed in users' prosthetic socket in response to changes in their limb volume was assessed.

This paper presents a preliminary study that was carried out in two phases. The first phase, the three-ply phase, was conducted to demonstrate the feasibility of the proposed method. In this regard, variations of three sock plies (determined by a collaborating prosthetist) were tested with respect to the volume of the limb. In this phase, the locations at the bottom of the socket at which the variations of force would be representative of the changes in the volume of the limb were identified. Findings of this experiment motivated proceeding to the second phase of the study, the one-ply phase, which was carried out to determine if smaller variations in the volume, simulated by variations of one or two sock plies, could be detected using the proposed method.

In both phases, data from FSRs embedded inside the users' sockets were collected as they walked a marked distance. Collected data were then analyzed offline to determine if signals acquired from the sensory system could be used to determine variations in the volume of the limb.

Experiments conducted in this study were approved by the Office of Research Ethics at Simon Fraser University, and informed consents were provided by participants. All experiments were conducted under supervision of the Certified Prosthetist who collaborated in this study. 


\subsection{Participants}

In the three-ply phase of this study, data were collected from four participants with transtibial amputations. As the one-ply phase was carried out based on and motivated by results of the three-ply phase, participant recruitment for this phase included participants of the three-ply phase. We were only able to recruit three new participants with transtibial amputations for this phase.

All participants used their prosthesis on a daily basis. Information about participants' weight, cause of amputation, and prosthesis can be found in Table 1.

Table 1. Summary of participants' information. Prosthesis: Total Surface Bearing (TSB), Thermoplastic Elastomer (TPE), Patellar Tendon Bearing (PTB).

\begin{tabular}{cccccc}
\hline Phase & Participant & Amputation Side & Cause & Weight Range (kg) & Prosthesis \\
\hline $\begin{array}{c}\text { Three-ply } \\
\text { Phase }\end{array}$ & 1 & Right & Trauma & $65-70$ & $\begin{array}{c}\text { TSB socket with a TPE cushion } \\
\text { liner and a suspension sleeve }\end{array}$ \\
& 2 & Left & Trauma & $85-90$ & $\begin{array}{c}\text { PTB socket with a sock fit and } \\
\text { sleeve suspension }\end{array}$ \\
$\begin{array}{c}\text { One-ply } \\
\text { Phase }\end{array}$ & 1 & Left & Vascular & $90-95$ & $\begin{array}{c}\text { TSB soct with a hybrid } \\
\text { locking liner }\end{array}$ \\
& 2 & Right & Trauma & $110-115$ & $\begin{array}{c}\text { TSB socket with a silicone cushion } \\
\text { liner and a sleeve suspension }\end{array}$ \\
& 2 & Right & Trauma & $100-105$ & $\begin{array}{c}\text { TSB socket with a hybrid locking liner } \\
\text { Hybrid socket with a gel sock } \\
\text { and sleeve suspension }\end{array}$ \\
& 3 & Right & Trauma & $80-85$ & $\begin{array}{c}\text { TSB socket with a silicone cushion } \\
\text { liner and sleeve suspension }\end{array}$ \\
\hline
\end{tabular}

Volunteers were recruited by Barber Prosthetics Clinic (BPC) (Vancouver, BC, Canada). Individuals that participated in the study had unilateral transtibial amputations, were $18+$ years old, wore three or more plies of prosthetic socks, and did not present any cognitive impairments.

\subsection{Hardware}

The sensory systems used for data collection in this study included FSR sensors from Tangio Printed Electronics (North Vancouver, Canada), model TPE-502 round sensors with the diameter of approximately $2 \mathrm{~cm}$. FSRs were connected to an Arduino model Pro Mini that would register their responses through a voltage divider circuit where the FSR sensors were connected between $V_{d d}$-that was provided by the Arduino-and $V_{\text {out }}$, and a base resistor $\left(327 \Omega\right.$ ) was connected between $V_{\text {out }}$ and ground. All sensors were connected to the same resistor on one side, and a separate Arduino output on the other side. The voltage at the Arduino side of each sensor was raised to $V_{d d}$ when the other sensors were not supplied with a voltage. As a result, only one sensor was activated at each point in time. Analog sensor readings obtained through this method were converted to digital values to be read by the Arduino. Sensor readings were transferred to a computer by the Arduino board wirelessly using a Bluetooth module. The system was powered using a 3.7 V Lithium Polymer (LiPo) battery. The main components of the system are shown in Figure 1.

Placement of the sensors used in the three-ply phase was done to ensure coverage of the main areas from which we expected to be able to retrieve information about fluctuations in the volume of the residual limb. In this phase, four sensors were placed in the distal, anterior distal, medial distal, and lateral distal locations inside the prosthetic sockets that participants used on a daily basis. The distance between locations of the anterior distal, medial distal, and lateral distal sensors from the distal end of the socket was approximately $6 \mathrm{~cm}$. The distal end of the socket was selected since it was expected that an increase in the volume of the residual limb would cause the limb to be pushed upward inside the socket. This would lead to a decrease in the interface pressure at the distal end of the socket. This hypothesis was based on the literature and the experience of the collaborating prosthetist [22]. The other three locations were also selected based on evidence from the literature and 
the expertise of the collaborating prosthetist [23]. These locations were expected to experience greater pressure/force changes as they were areas of the limb with bony prominences. Posterior distal pressure was not considered as less pressure variation was expected at this location compared to the other distal locations due to higher ratios of soft tissue at this location in most individuals. Placement of sensors inside the prosthetic socket in the three-ply phase is shown in Figure 1. Out of the four positions that were tested in the three-ply phase, the distal location was selected and the other three were removed form experiments of the one-ply phase based on results of the three-ply phase.

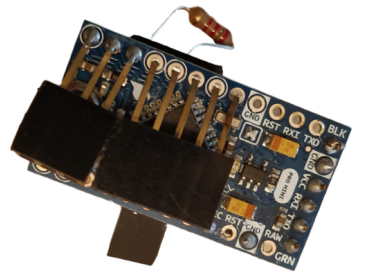

(a)

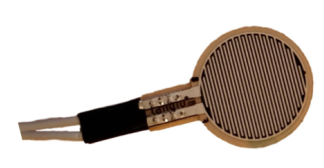

(c)

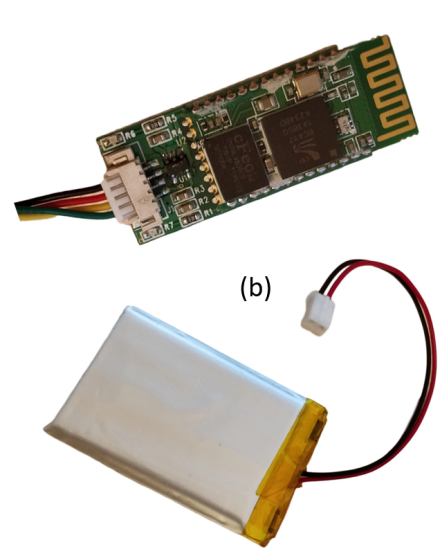

(d)

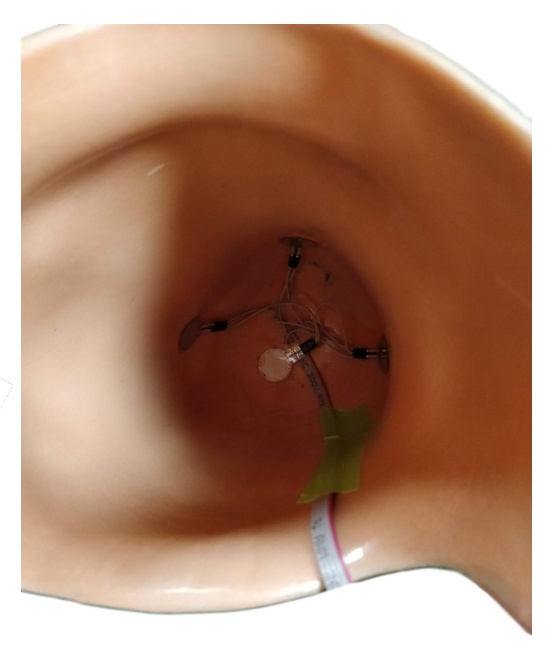

Figure 1. The hardware setup. The figure on the left shows the main components of the system: (a) an Arduino Pro Mini board, (b) a Bluetooth module, (c) a force sensitive resistor, (d) a lithium polymer battery. The figure on the right shows the placement of the four force sensitive resistors (FSRs) inside the socket in the distal, anterior distal, lateral distal, and medial distal locations. The image shows the lower limb prosthetic socket from the top. The bottom of the image shows the posterior side of the socket.

\subsection{Protocol}

To collect data, FSRs were placed inside participants' sockets. This was performed in the beginning of the data collection session for each participant. The locations of sensors were not modified throughout the session.

In the experiments conducted for both phases of the study, prosthetic socks were used to simulate variations in the volume of the limb. Prosthetic socks were added to increase the volume; conversely, socks were removed to reduce the volume. Three sock ply conditions were considered in both phases: 'normal plies', 'less plies', and 'extra plies'. In both phases, the number of sock plies that was used in the normal plies

\subsubsection{Three-Ply Phase}

For this phase, in the less plies condition, the participants wore three sock plies less compared to the normal plies condition, and in the extra plies condition, they wore three plies more than the normal plies condition.

\subsubsection{One-Ply Phase}

In the second phase, each condition differed from the next/previous one by variation of one sock ply. To reduce the effect of differences in the thicknesses of sock plies due to the sock's age, manufacturer, etc., in each condition, a one-ply sock was either added or removed to ensure the increase/decrease in the thickness. 
Between different sock ply conditions, the participant doffed their prosthetic socket to adjust the number of sock plies worn on their limb based on the condition being investigated. After adjustment of sock plies, the participant donned their prosthesis without any further preparation and performed the protocol of the test. The protocol consisted of the participants walking a marked distance of approximately three meters on a flat surface back and forth. Five repetitions of this was performed for each sock ply condition. FSR signals and videos were recorded as participants were performing the task of the protocol. Videos and FSR signals were acquired using Labview and were synchronized using their recorded timing.

\subsection{Data Analysis}

To separate sections of data corresponding to the time volunteers were performing the walking task of the protocol, recorded videos were used. Labeling of data according to video sections was performed manually for each individual and each sock ply condition. Raw FSR signals were filtered using a second order Butterworth low pass filter to remove noise. The cut-off frequency for the Butterworth filter was determined based on the Welch's power spectral density estimate of the signal being filtered. Peaks of filtered signals were extracted. Each peak corresponded to one step as pressure/force at the distal end of the socket peaks once in every step taken by the monitored foot, the leg with amputation in this case, at the time when the maximum amount of user's weight is loaded on the foot during the mid-stance phase of a gait cycle. Peak values of FSR signals were averaged to obtain a single value per sensor for each participant and each condition. This averaging step was required because peak values were dependent and treating them as separate measurements would have caused pseudoreplication [24]. Moreover, averaging multiple steps for each participant and each test provides a more realistic measure of the force by taking into account the variability of gait in different steps.

The average peak values of FSR readings were converted to force. To convert FSR readings to force, a calibration method was used in which the sensors' readings in response to known values of varying applied force were measured for multiple FSR sensors. In this experiment, the value of force was measured using an ATI Mini 45 six-axis load cell as the force was increased up to approximately $280 \mathrm{~N}$ and decreased in cycles of approximately $25 \mathrm{~s}$. Using measurements obtained in this experiment, an exponential curve was fitted to map the sensors' responses to force values $\left(R^{2}=0.944, R M S E \%=1.85 \%\right)$. Exponential fitting was chosen for this calibration based on the documentation of FSR sensors used in this study.

In order to generalize the results, average peaks of force values were normalized with respect to the values obtained in the normal plies condition for each participant. Normalized means of peaks of force were final measures compared in different conditions of sock plies. The process of data analysis is shown in Figure 2.

It is worth noting that at the beginning of each test, after donning the socket, there was a transient period when the user's residual limb and the prosthetic socket were adjusting to the test conditions. The length of this time in each test depended on the socket fit, the shape of the residual limb, the number of sock plies, and other variables such as the user's weight. For the purpose of this study, this transient section, which was four steps on average, was removed from the beginning of the protocol in each test condition since it appeared only at the beginning of the tests and did not represent normal operations of the socket.

For statistical analysis, a randomized complete block experimental design with mixed-model analysis was used where volunteers were considered random effects. This was done to remove the effect of variations of data due to individual-specific factors [25]. In this repeated measures design, normalized means of peaks of force obtained from different participants for each sock ply condition and for each phase were considered as repeated measures. All statistical analyses performed for this study were done using JMP statistical analysis software from the Statistical Analysis System (SAS) institute (Cary, NC, USA). 
Statistical analysis was performed based on the assumption of normality of residuals. Shapiro-Wilk test, which is recommended for small sample sizes [26], was used to assure normality of residuals before statistical tests were carried out in this study.

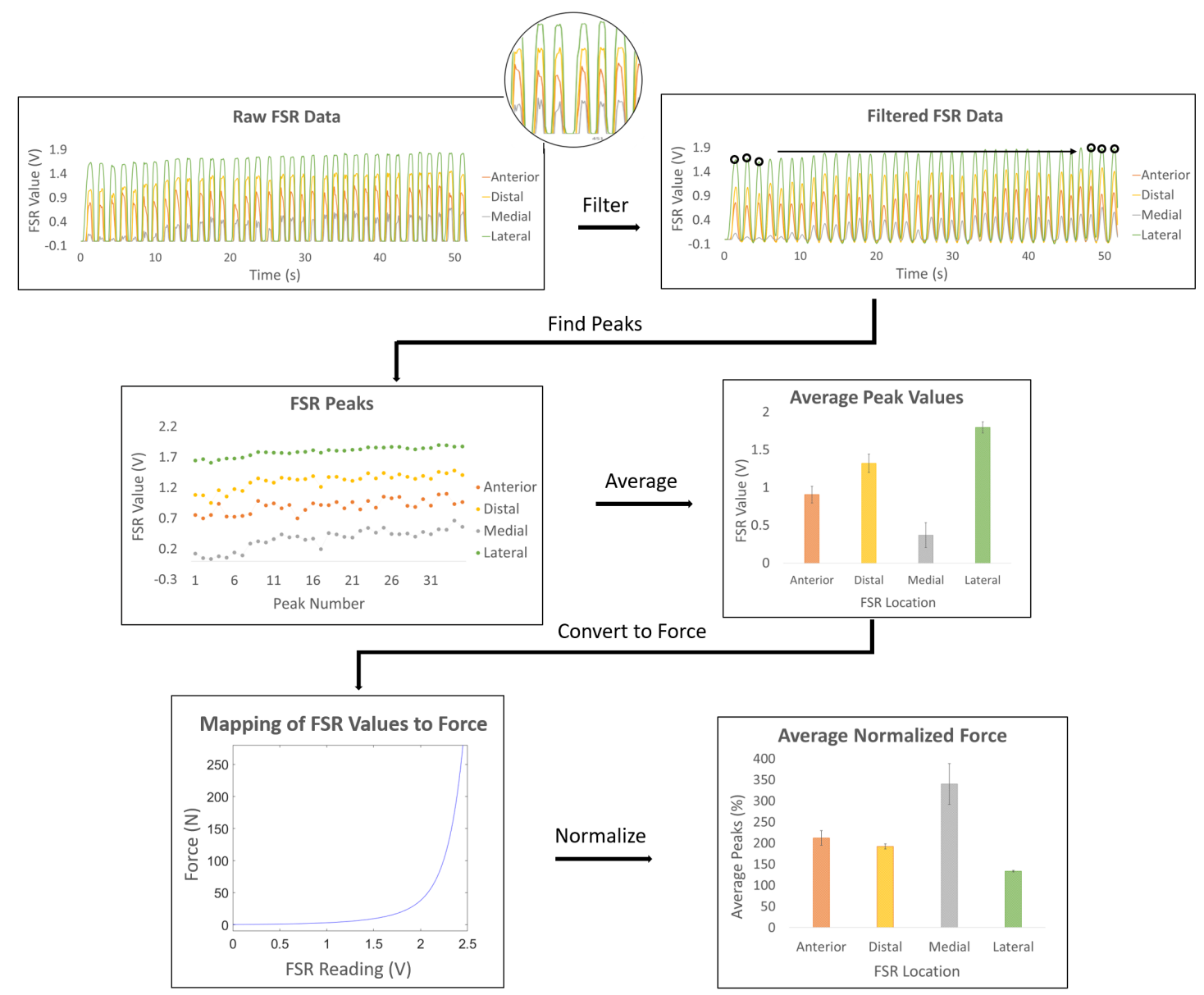

Figure 2. The data analysis process. The data analysis process consisted of filtering raw force sensitive resistor (FSR) signals, finding peaks of the filtered signals, averaging peak values, converting average values to force, and normalizing the average peaks of force.

\section{Results}

\subsection{Three-Ply Phase}

Results of the three-ply phase showed that for all participants, the force measured by the sensor placed at the distal location decreased as the volume increased. This observation is consistent with the expected pattern of variations in the interface force as a result of fluctuations in the volume of the residual limb. Normalized means of peaks of force obtained at the distal location for the four participants of the three-ply phase are shown in Figure 3.

In two other considered locations, namely the lateral distal and the anterior distal locations, the force measured by sensors followed the expected pattern in all participants except in participant 2 . The normalized average peak of force at the medial distal location did not follow the expected pattern in either participant 2 or participant 3. Moving forward with this study, we chose to use the distal location as it was consistent in all participants. 


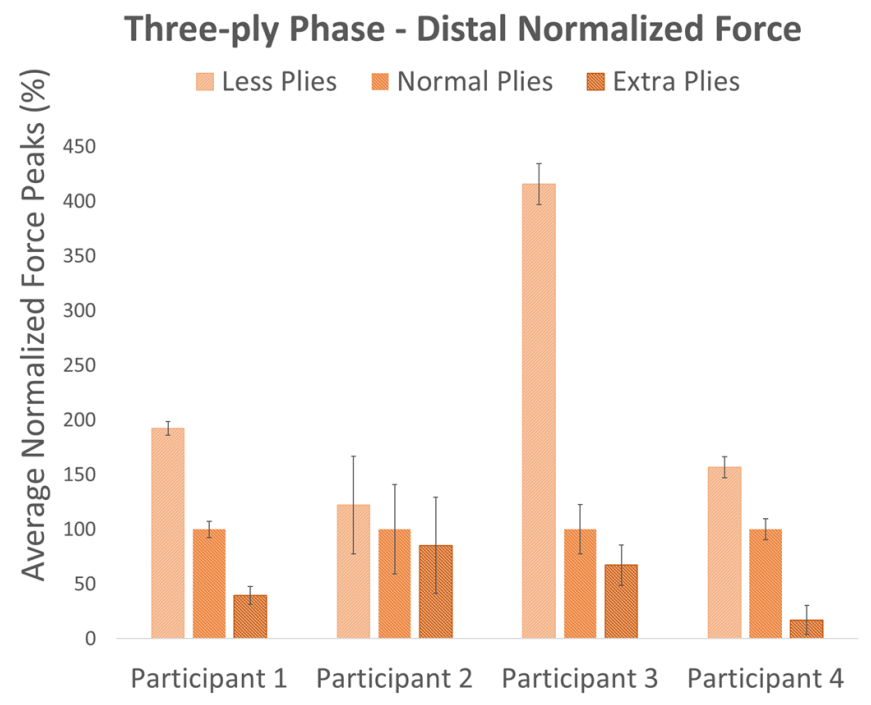

Figure 3. Results of the three-ply phase. Average peaks of normalized force in the distal location of the prosthetic sockets of the four participants in the three-ply phase.

To determine if the normalized mean of distal force peaks changed significantly in response to variations in the volume of the limb, mixed-model analysis was performed on the results of the three-ply phase. In this analysis, the null hypothesis was considered to be the normalized mean of distal force peaks remaining the same in different sock ply conditions. As a result, rejecting the null hypothesis would determine that the normalized mean of distal force peaks changed in response to variations in the volume of the limb, thus proving that there is a relationship between distal force and volume of the limb. This confirms that volume variations can be determined by monitoring the distal force.

Results of the Shapiro-Wilk test determined normality of residuals (less plies: $p=0.1415$ and extra plies: $p=0.8833$ ) confirming that assumption of the statistical tests used in this study were not violated. It should be noted that all values for the normal plies condition were similar $(100 \%)$ due to the normalization step. Results of mixed model analysis rejected the null hypothesis $(\mathrm{F}(2,6)=5.1742, p=0.0494)$, proving the feasibility of our proposed method.

Results of the two phases are also shown in Figure 4. This figure shows adjusted means and standard errors as predicted by the model fit to data from all individuals, as repeated measures, of each phase during mixed model analysis [27]. Using the adjusted means of the different sock ply conditions was deemed appropriate for quantitative representation of the results of this study since it isolated the effect of sock plies by eliminating the effect of individual-specific variables, such as body weight and socket fit. 
Adjusted Means of Sock Plies Effect Three-ply Phase

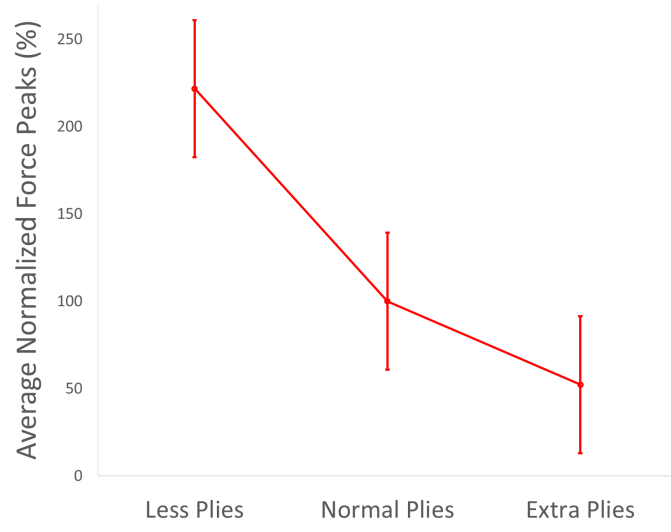

Adjusted Means of Sock Plies Effect One-ply Phase

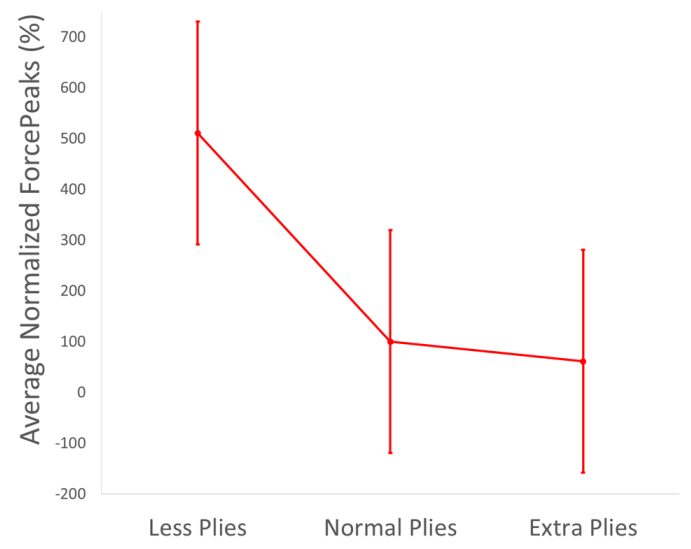

Figure 4. Results in terms of adjusted means and standard errors. Adjusted normalized means of peaks of force in the distal location across participants in the two phases of the study.

\subsection{One-Ply Phase}

In this phase, normalized means of force peaks at the location that was determined to be effective in the three-ply phase, i.e., the distal location, were compared in three conditions varying by one prosthetic sock ply. To determine the effect of two-sock-ply increments, the less plies and extra plies conditions that varied in the number of sock plies by two were also compared. Figures 4 and 5 show results of both one-ply and two-ply variations.

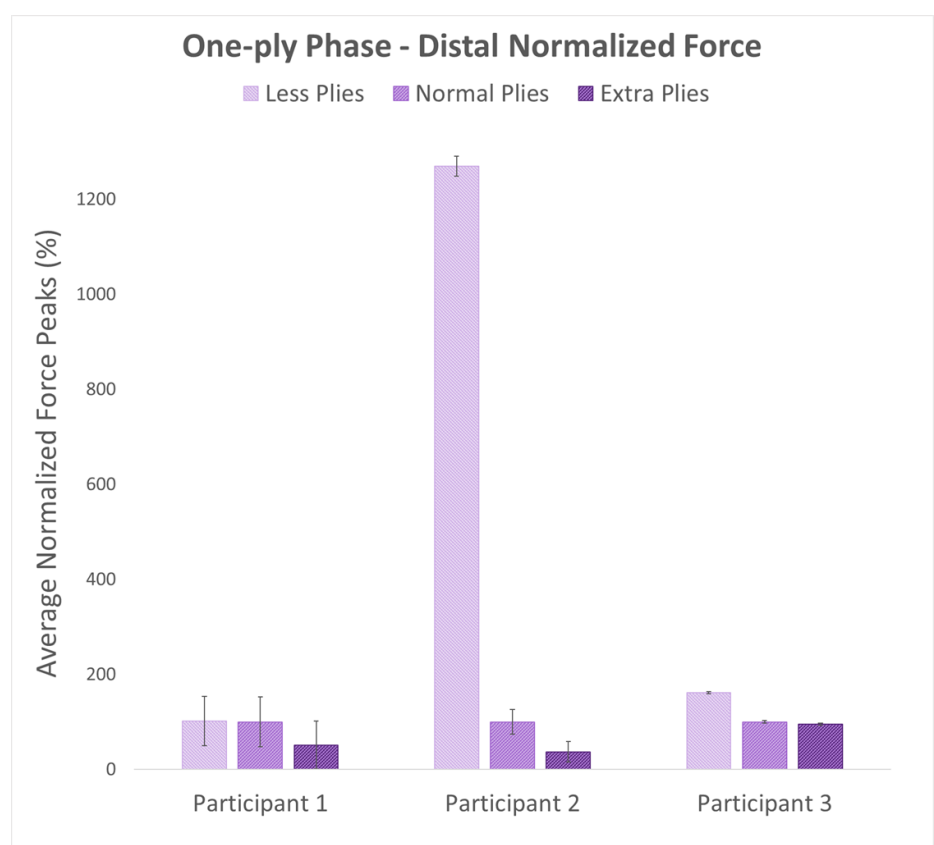

Figure 5. Results of the one-ply phase. Normalized average peaks of force in the distal location of the prosthetic sockets of the three participants in the one-ply phase.

As volume increased by either one ply or two plies, the normalized average peak of force decreased in this location for all participants. This was the expected pattern and was consistent with the results of the first phase of the study. However, future studies with more participants are required to confirm results of this phase with statistical analysis. Adjusted normalized means across participants are shown in Figure 4. 


\section{Discussion}

An existing method that can be used to assist individuals with amputations with the management of their residual limbs' volume fluctuations is based on measurements of the volume of the limb. Bioimpedence is the most practical volume measurement technique for this purpose. Compared to the proposed method of this study, the disadvantage of bioimpedence is that it requires proper electrical contact between electrodes and the skin through the use of hydrogel. Moreover, this method is dependent on the cause of volume fluctuations as it measures variations in the fluid volume of the limb. Another existing method that can be utilized towards the goal of this study is to use the limb-socket interface pressure registered using load cells. The main disadvantages of this method compared to the one proposed in this paper are: it requires alterations of the prosthetic socket for sensor mounts; it increases the weight of the prosthetic system; and it is relatively expensive. Alternatively, the pressure map at the interface of the residual limb and the prosthetic socket can be obtained using computational methods. The con of this method compared to that of this study is that it requires precise measurements for each individual derived from scans of the limb. This makes the computational method relatively complex and expensive.

The method proposed in this study is novel since, unlike most other methods, it is easily integrated within the user's prosthesis with minimal modifications. The sensory system requires one time installation and can be used seamlessly afterwards. Using this method, volume variations can be detected as the user carries out their daily activities using the prosthetic socket without a need for doffing the socket. This method is simple to use and does not require extensive calibration before use. Signals used in this method can be potentially acquired throughout extended periods of time. FSR signals are easy to process, making this method computationally efficient [28,29]. Another advantage of this method is that it does not require direct contact with the skin and is less prone to errors due to environmental changes compared to some of the alternative methods. Moreover, this method, unlike some alternatives, does not depend on the reason of volume change. For instance, bioimpedance measures variations in the fluid volume of the limb rather than directly measuring the volume of the limb itself. One potential application of the proposed method is developing a smart volume adjustment system, e.g., using adjustable bladders inside prosthetic sockets [30].

The hypothesis used in this study, which guided the design choices such as the number and placement of the sensors, was based on the literature and the expertise of the Certified Prosthetist who collaborated in this work. Sanders et al. conducted a study that monitored distal positioning of the residual limb inside a prosthetic socket. In this study, they reported that a larger volume of the residual limb would cause a more proximal placement inside the socket, relieving some of the distal pressure [22]. Based on the results reported by Sanders et al. and the experience of the collaborating Certified Prosthetist, it was clear that the interface pressure at the distal location would provide information about fluctuations in the volume of the residual limb. Although the pressure in other areas of the limb-socket interface would also be affected by variations in the limb's volume, the distal pressure was expected to be more consistent amongst different individuals.

A possible approach to investigate the feasibility of the proposed method could be to increase the number of sensors used in the three-ply phase. However, we used four sensors as it was already clear that the distal location was the most promising location for the goal of this study. The reason for placing three other sensors in the anterior distal, medial distal, and lateral distal locations was to ensure coverage of the main areas close to the one from which we expected to be able to retrieve information about fluctuations in the volume of the residual limb. Moreover, we decided to carry out the experiments with a limited number of sensors to maintain a simple setup and limit the amount of wiring to ensure the practicality of running the test with participants. Although more sensors could be used in preliminary tests and for short term solutions, there were requests from clinicians to minimize the number of sensors to assure practicality of using the method in long term applications of the system. The experimental results of our work confirmed our hypothesis regarding the number and placement of the sensors. 
Although the labeling of data was performed manually based on video recordings, this procedure was accurate as the beginning and the end of each step were confirmed with the FSR-sensor data. The manual labeling was performed only to remove the steps performed by the users to turn around in between repetitions. Moreover, small errors in labeling would cause missing FSR data in the beginning of the first step or at the end of the last step of a repetition which would not affect the result since the outcome measure was based on the peak of FSR values which occurred mid-step. As a result, we believe that manual labeling of data has not affected the results obtained in this study.

The reason that the force at the anterior distal, medial distal, and lateral distal locations did not follow the expected pattern in the three-ply phase might be that for those locations, sensors were placed more proximally compared to the distal location. This makes these locations more prone to variations in interface pressure that are caused by user's socket fit, style of socket, and movements of the limb inside the socket. For example, depending on the shape of the residual limb and the relative location of the Tibia bone, the pressure distribution at the anterior location could vary in different individuals. On the other hand, all users were using non-distal-bearing sockets which made the pressure at the distal location more consistent. Moreover, an increase in the volume of the limb results in an increased width/diameter of the limb which could affect the more proximal locations.

Results obtained for participant 2 in the one-ply phase demonstrate high inter-participant variability between this individual and others in this phase. Inter-participant variability in the distal pressure can be caused by multiple factors such as the user's wight, the shape and the type of the socket, the socket fit, and the shape of the residual limb in addition to whether prosthetic liners are used or not. Participant 2 of the second phase used a gel sock instead of a prosthetic liner which, in addition to other differing factors including the type of the socket and the shape of the limb, has likely influenced this participant's measurements. Another reason for such measurement differences is possibly the fact that this participant had suffered a foot injury in their intact limb a few months prior to the experiment. Nevertheless, the pattern of variation in the distal force remained as expected for this participant. This demonstrates that the distal pressure decreases with an increase in the volume of the limb regardless of individual-specific factors mentioned above.

Although force measurements in three out of the four locations tested in the three-ply phase did not demonstrate the expected pattern of variation in response to changes in the volume of the limb, noting that the initial hypothesis of this study was based on distal force, results of the three-ply phase confirm this hypothesis. It should be noted that the other three sensors in the three-ply phase were only added to ensure coverage of the main areas that were close to the distal location and were expected to experience greater pressure/force changes. It should also be noted that sample sizes used in this preliminary study were small and further studies with larger sample sizes are needed to confirm the results of this work.

Although the pattern of variations in the distal normalized force peaks was consistent among participants, the absolute value of this measure or the amount of reduction in it with an increase of three sock plies was not the same in all participants. The high inter-participant variability of force measurements is likely due to factors such as user's body weight and their socket fit. As also reported in other studies [31], variability in such participant-specific factors affects the distribution and magnitude of socket-limb interface force/pressure. For example, the load supported by the prosthetic socket would be higher for an individual with higher body weight. This would lead to a higher total socket-limb interface pressure.

This study did not identify a consistent relationship between the number of sock plies and the distal force. The results showed that there is a monotonic relationship, but it is individual-dependent as there is a high inter-participant variability in force measurements. In addition, the fact that each amputation and each socket are different from the other makes it difficult to generalize the results with a specific relationship at this point. In fact, as shown in Figure 5, adding one sock ply in one individual has a different effect compared to adding the same number of plies to another individual. Information from a larger population including a number of parameters such as body weight, 
circumference of the limb, height, location of amputation, shape of the socket, and others in future work may lead to the identification of a relationship that can be generalized across multiple individuals.

The small sample sizes used in the two phases of this study are amongst its limitations and lead to low powers of statistical tests, resulting in higher probabilities of making type II errors. However, when significance is determined, one does not need to worry about type II errors as they only occur when significance cannot be determined [32]. The $p$-value obtained in the statistical analysis performed in this phase was marginal $(p=0.049)$ which might be due to the high probability of type II errors which prevent rejection of the null hypothesis when it is false. Results of this analysis should be confirmed in future studies with larger sample sizes.

As a result that the significance was not determined in the one-ply phase, statistical analysis was not valid for this phase as its results might have been compromised by type II errors. Thus, results of statistical analysis of the one-ply phase were not reported. Results of the one-ply phase should only be considered quantitatively and need to be confirmed by future studies with larger sample sizes.

Although more accurate types of pressure/force sensors, such as load cells, could be used towards the goal of this study, these sensors would result in a costly system which was not aligned with the objective of this work. As confirmed by results reported in the manuscript, FSRs, despite their non-linear response and their inferior accuracy of measurement compared to load cells, can be an inexpensive alternative to such sensors and can provide measurements to determine variations in the volume of the residual limb.

To use the proposed method in practice, the sensory system should be embedded in the prosthetic socket of users, and data should be recorded continuously throughout the day. In this scenario, an algorithm would be required to detect the walking action during which FSR signals would be used to determine variations in the residual limb's volume. Various methods can be used for detection of the walking action, one of which is the use of classification algorithms.

In the case of applications for individuals with disabilities, case-by-case variability is an important factor and should be considered. The proposed method of this study was tested using different types of sockets as shown in Table 1. Two of the sockets had cut outs at the distal end to allow for the pin attached to locking liners. In this study, there were also variations in the types of the liners used. For instance, one of the participants did not use any liners, and another one used a sheath instead of a liner while others used silicone or thermoplastic elastomer (TPE) liners. Moreover, there were variations in weight, age, amount of experience in using a prosthetic socket, and other factors in participants of the study. Despite these case-by-case variations in the participants and the devices used by them, a similar trend of variation in sensor readings as a result of changes in the volume of the residual limb was determined. From results of this study, we believe that the proposed method is not sensitive to such case-by-case variations and that the same method could be used to achieve the goal regardless of such variations.

The main limitation of the work presented in this study is the small samples sizes used in the experiments of the two phases. Moreover, due to the preliminary nature of this work, integration of attained information with a feedback system was not considered.

\section{Conclusions}

With the goal of assisting users with the management of their volume adjustment system, this study proposed a method to determine variations in the volume of the limb by monitoring the force at the bottom of the lower extremity socket as the user walked. Results showed that a simple hardware setup with a single FSR sensor can extract information about the fluctuations in the volume of the limb. However, determining the smallest detectable variation in the volume with this technique requires a larger number of participants and more extensive experiments. The proposed method can potentially be used to provide real-time feedback to users to assist them with the management of their volume adjustment system, e.g., sock plies, and help them maintain a good prosthetic socket fit to avoid skin problems such as ulcers. We believe that the simple system proposed in this work can be 
translated into a technology which can be commercialized and have a tremendous impact on the lives of individuals with amputations in the short term.

\section{Limitations and Future Work}

One of the limitations of this preliminary study was the limited number of participants in the two experiments. The reason for this limitation was the difficulty of recruiting participants with amputations. Moving forward, experiments with a larger number of participants should be conducted to determine the smallest detectable variation in the volume of the limb by the proposed method. A larger population could further increase the confidence level in relation to the possibility of detecting variations in the volume of the limb.

The system proposed in this study was developed with the primary goal of providing feedback to the users for their limbs' volume adjustments to maintain a high quality of socket fit. To achieve this, a feedback system needs to be incorporated into the system in future work that alerts users when their limb volume needs to be adjusted, e.g., by adding or removing prosthetic socks. Such a feedback can be provided through a mobile application that connects to the sensory system. An added feature to this application could be the continuous calibration of the system.

On average, the five repetitions of each test took approximately one minute for each participant. Prosthetic sockets were then doffed to adjust the number of sock plies, also releasing any pressure from FSRs between tests in the meanwhile. Since these tests were carried out in a short amount of time, we did not take the drift, creep, or the effect of temperature changes into account. The effect of these factors in prolonged use of the sensors should be characterized and accounted for to make the long-term application of the system possible.

Author Contributions: Conceptualization, C.A., B.P., and C.M.; methodology, C.A. and B.P.; validation, C.A. and C.M.; formal analysis, C.A.; investigation, C.A.; writing-original draft preparation, C.A.; writing-review and editing, B.P. and C.M.; visualization, C.A.; supervision, C.M.; project administration, C.M.; funding acquisition, C.M. All authors have read and agreed to the published version of the manuscript.

Funding: Research supported by the Natural Sciences and Engineering Research Council of Canada (NSERC), the Canadian Institutes of Health Research (CIHR), and the Canada Research Chair (CRC) program.

Conflicts of Interest: The Principal Investigator, Carlo Menon, and members of his research team have a vested interest in commercializing the technology tested in this study, if it is proven to be successful and may benefit financially from its potential commercialization.

\section{Abbreviations \\ $\begin{array}{ll}\text { FSR } & \text { Force Sensitive Resistor } \\ \text { TSB } & \text { Total Surface Bearing } \\ \text { TPE } & \text { Thermoplastic Elastomer } \\ \text { PTB } & \text { Patellar Tendon Bearing } \\ \text { HMI } & \text { Human-Machine Interface }\end{array}$}

The following abbreviations are used in this manuscript:

\section{References}

1. Tantua, A.T.; Geertzen, J.H.; van den Dungen, J.J.; Breek, J.K.C.; Dijkstra, P.U. Reduction of residual limb volume in people with transtibial amputation. J. Rehabil. Res. Dev. 2014, 51, 1119-1126. [CrossRef] [PubMed]

2. Sanders, J.E.; Fatone, S. Residual limb volume change: Systematic review of measurement and management. J. Rehabil. Res. Dev. 2011, 48, 949-986. [CrossRef] [PubMed]

3. Sanders, J.E.; Harrison, D.S.; Allyn, K.J.; Myers, T.R. Clinical utility of in-socket residual limb volume change measurement: Case study results. Prosthet. Orthot. Int. 2009, 33, 378-390. [CrossRef] [PubMed]

4. D'Silva, K.; Hafner, B.J.; Allyn, K.J.; Sanders, J.E. Self-reported prosthetic sock use among persons with transtibial amputation. Prosthet. Orthot. Int. 2014, 38, 321-331. [CrossRef] [PubMed] 
5. National Academies of Sciences, Engineering, and Medicine. The Promise of Assistive Technology to Enhance Activity and Work Participation; National Academies Press: Washington, DC, USA, 2017.

6. Ziegler-Graham, K.; MacKenzie, E.J.; Ephraim, P.L.; Travison, T.G.; Brookmeyer, R. Estimating the Prevalence of Limb Loss in the United States: 2005 to 2050. Arch. Phys. Med. Rehabil. 2008, 89, 422-429. [CrossRef]

7. Ahmadizadeh, C.; Menon, C. Investigation of Regression Methods for Reduction of Errors Caused by Bending of FSR-Based Pressure Sensing Systems Used for Prosthetic Applications. Sensors 2019, 19, 5519. [CrossRef]

8. Lingala, S.M.; Ghany, M.G.M.M. How do sock ply changes affect residual limb fluid volume in people with trans-tibial amputation? J. Rehabil. Res. Dev. 2016, 25, 289-313. [CrossRef]

9. Staker, M.; Ryan, K.; LaBat, K. Medicine and design investigate residual limb volume fluctuations: Three case studies. Australas. Med. J. 2009, 2, 156-161. [CrossRef]

10. Greenwald, R.M.; Dean, R.C.; Wayne, J. Volume Management: Smart Variable Geometry Socket (SVGS) Technology for Lower-Limb Prostheses. J. Prosthet. Orthot. 2003, 15, 107-112. [CrossRef]

11. Sanders, J.E.; Murthy, R.; Cagle, J.C.; Allyn, K.J.; Phillips, R.H.; Otis, B.P. A Device to monitor sock use in people using prosthetic limbs: Technical report. J. Rehabil. Res. Dev. 2012, 49, 1229-1238. [CrossRef]

12. Sanders, J.E.; Rogers, E.L.; Abrahamson, D.C. Assessment of residual-limb volume change using bioimpedence. J. Rehabil. Res. Dev. 2007, 44, 525-535. [CrossRef] [PubMed]

13. Sanders, J.E.; Moehring, M.A.; Rothlisberger, T.M.; Phillips, R.H.; Hartley, T.; Dietrich, C.R.; Redd, C.B.; Gardner, D.W.; Cagle, J.C. A bioimpedance analysis platform for amputee residual limb assessment. IEEE Trans. Biomed. Eng. 2016, 63, 1760-1770. [CrossRef] [PubMed]

14. Rinaudo, A.; Raffa, G.M.; Scardulla, F.; Pilato, M.; Scardulla, C.; Pasta, S. Biomechanical implications of excessive endograft protrusion into the aortic arch after thoracic endovascular repair. Comput. Biol. Med. 2015, 66, 235-241. [CrossRef]

15. Gallo, A.; Agnese, V.; Coronnello, C.; Raffa, G.M.; Bellavia, D.; Conaldi, P.G.; Pilato, M.; Pasta, S. On the prospect of serum exosomal miRNA profiling and protein biomarkers for the diagnosis of ascending aortic dilatation in patients with bicuspid and tricuspid aortic valve. Int. J. Cardiol. 2018, 273, 230-236. [CrossRef]

16. Sengeh, D.M.; Moerman, K.M.; Petron, A.; Herr, H. Multi-material 3-D viscoelastic model of a transtibial residuum from in-vivo indentation and MRI data. J. Mech. Behav. Biomed. Mater. 2016, 59, 379-392. [CrossRef] [PubMed]

17. Sanders, J.; Zachariah, S.; Jacobsen, A.; Fergason, J. Changes in interface pressures and shear stresses over time on trans-tibial amputee subjects ambulating with prosthetic limbs: Comparison of diurnal and six-month differences. J. Biomech. 2005, 38, 1566-1573. [CrossRef] [PubMed]

18. Portnoy, S.; Yarnitzky, G.; Yizhar, Z.; Kristal, A.; Oppenheim, U.; Siev-Ner, I.; Gefen, A. Real-time patient-specific finite element analysis of internal stresses in the soft tissues of a residual limb: A new tool for prosthetic fitting. Ann. Biomed. Eng. 2007, 35, 120-135. [CrossRef]

19. Mak, A.F.; Zhang, M.; Boone, D.A. State-of-the-art research in lower-limb prosthetic biomechanics-socket interface: A review. J. Rehabil. Res. Dev. 2001, 38, 161-74. [PubMed]

20. Sengeh, D.M.; Herr, H. A Variable-Impedance Prosthetic Socket for a Transtibial Amputee Designed from Magnetic Resonance Imaging Data. J. Prosthet. Orthot. 2013, 25, 129-137. [CrossRef]

21. Sanders, J.; Jacobsen, A.; Fergason, J. Effects of fluid insert volume changes on socket pressures and shear stresses: Case studies from two trans-tibial amputee subjects. Prosthet. Orthot. Int. 2006, 30, 257-269. [CrossRef]

22. Sanders, J.E.; Karchin, A.; Fergason, J.R.; Sorenson, E.A. A noncontact sensor for measurement of distal residual-limb position during walking. J. Rehabil. Res. Dev. 2006, 43, 509. [CrossRef] [PubMed]

23. Sanders, J.; Greve, J.; Clinton, C.; Hafner, B. Clinical study: Changes in interface pressure and stump shape over time: Preliminary results from a trans-tibial amputee subject. Prosthet. Orthot. Int. 2000, 24, 163-168. [CrossRef] [PubMed]

24. Heffner, R.A.; Butler, M.J.; Reilly, C.K. Pseudoreplication revisited. Ecology 1996, 77, 2558-2562. [CrossRef]

25. Yang, R.C. Towards understanding and use of mixed-model analysis of agricultural experiments. Can. J. Plant Sci. 2010, 90, 605-627. [CrossRef]

26. Ghasemi, A.; Zahediasl, S. Normality tests for statistical analysis: A guide for non-statisticians. Int. J. Endocrinol. Metab. 2012, 10, 486. [CrossRef] 
27. Sall, J.; Stephens, M.L.; Lehman, A.; Loring, S. JMP Start Statistics: A Guide to Statistics and Data Analysis Using JMP; Sas Institute: Cary, NC, USA, 2017.

28. Ahmadizadeh, C.; Menon, C.; Pousett, B.; Menon, C. Investigation of Feature Selection for Gesture Classification for Prosthesis Control Using Force Myography: A Case Study. Front. Bioeng. Biotechnol. 2019, 7, 331. [CrossRef]

29. Belyea, A.T.; Englehart, K.B. A proportional control scheme for high density force myography. J. Neural Eng. 2018, 15, 046029. [CrossRef]

30. Sanders, J.E.; Redd, C.B.; Cagle, J.C.; Hafner, B.J.; Gardner, D.; Allyn, K.J.; Harrison, D.S.; Ciol, M.A. Preliminary evaluation of a novel bladder-liner for facilitating residual-limb fluid volume recovery without doffing. J. Rehabil. Res. Dev. 2016, 53, 1107-1120.

31. Portnoy, S.; van Haare, J.; Geers, R.P.; Kristal, A.; Siev-Ner, I.; Seelen, H.A.; Oomens, C.W.; Gefen, A. Real-time subject-specific analyses of dynamic internal tissue loads in the residual limb of transtibial amputees. Med. Eng. Phys. 2010, 32, 312-323. [CrossRef]

32. Lieber, R.L. Statistical significance and statistical power in hypothesis testing. J. Orthop. Res. 1990, 8, $304-309$. [CrossRef]

(C) 2020 by the authors. Licensee MDPI, Basel, Switzerland. This article is an open access article distributed under the terms and conditions of the Creative Commons Attribution (CC BY) license (http://creativecommons.org/licenses/by/4.0/). 